\title{
Local jasmonic acid cues drive systemic acquired resistance signal generation
}

2

3 Jennifer Sales ${ }^{1}$, Elisabeth Pabst ${ }^{1}$, Marion Wenig ${ }^{1}$, Heiko H. Breitenbach ${ }^{1}$, Gerardo 4 Perez $^{1}$, Claudia Knappe ${ }^{1}$, Richard Hammerl², Jinghui Liu ${ }^{3}$, Wilfried Rozhon ${ }^{4}$, Brigitte

Poppenberger $^{4}$, Erwin Grill ${ }^{3}$, Corinna Dawid ${ }^{2}$, and A. Corina Vlot ${ }^{{ }^{*}}$

${ }^{1}$ Helmholtz Zentrum Muenchen, Department of Environmental Science, Institute of Biochemical Plant Pathology, Ingolstaedter Landstr. 1, 85764 Neuherberg, Germany; ${ }^{2}$ Technical University Munich, Chair of Food Chemistry and Molecular Sensory Science, Lise-Meitner-Str. 34, 85354 Freising; ${ }^{3}$ Technical University Munich, Chair of Botany, Emil-Ramann-Str. 4, 85354 Freising; ${ }^{4}$ Technical University Munich, TUM School of Life Sciences Weihenstephan, Biotechnology of Horticultural Crops, LieselBeckmann-Str. 1, 85354 Freising

*To whom correspondence should be addressed: corina.vlot@helmholtzmuenchen.de, $+49-89-31873985$

Running title: SA-JA cross talk drives systemic immunity

Key words: plant defence, systemic acquired resistance, salicylic acid, jasmonic acid, cross talk

Word counts:

Abstract: 148

Main text: 2999

Methods: 1495 


\section{Abstract}

2 The phytohormones salicylic acid (SA) and jasmonic acid (JA) promote two, mutually antagonistic immune pathways respectively protecting plants from biotrophic pathogens and necrotrophic pathogens or insects. This trade-off largely precludes the exploitation of SA and JA immune components for crop protection, raising the interest in immune signalling components that disrupt SA-JA antagonism. A local pathogen infection primes SA-dependent immunity in systemic tissues. This so-called systemic acquired resistance (SAR) ensures a long-lasting, broad-spectrum disease resistance that is not subject to SA-JA antagonism. Here, we show that two sequence-related LEGUME LECTIN-LIKE PROTEINs (LLPs) promote SAR through spatially separated functions with JA promoting local SAR signal generation through LLP3. In concert with LLP1, which is important for systemic recognition and propagation of SAR signals, LLP3 promotes both SA-dependent SAR and JAmediated immunity. Thus, exploitation of LLP-associated signalling cues might allow application of plant innate immune signals to promote (crop) plant health.

\section{Introduction}

As plants lack the dedicated immune cells and complex homeostatic systems that are found in animals, they developed alternative strategies of dealing with stress. An important aspect of this is the action of phytohormones and their associated signalling pathways. A key threat to plants comes from biotrophic or hemibiotrophic pathogens, which are fended off through salicylic acid (SA)-dependent responses induced at the site of infection. Pathogen-associated molecular patterns (PAMPs) from virulent pathogens are recognised by Pattern Recognition Receptors (PRRs) that are localised at the plasma membrane and initiate PAMP-triggered immunity $(\mathrm{PTI})^{1}$. Effectors from avirulent pathogens are recognised by intracellular nucleotidebinding domain and leucine-rich repeat proteins (NLRs), and initiate the relatively stronger effector-triggered immunity $(\mathrm{ETI})^{2}$. Both $\mathrm{PTI}$ and $\mathrm{ETI}$ responses rely on $\mathrm{SA}$. ENHANCED DISEASE SUSCEPTIBILITY1 (EDS1) acts as a central regulator upstream of SA driving a positive feedback loop with SA to fortify defence ${ }^{3,4}$. Once local SA cascades are triggered, a systemic signal is generated to upregulate defence in distal plant parts. This broad spectrum response is known as Systemic Acquired Resistance (SAR). EDS1 is required for a successful SAR response, as 
1 evidenced by defects in both SAR signal generation and recognition in eds 1 mutant 2 plants 5 .

3 During ETI, LEGUME LECTIN-LIKE PROTEIN1 (LLP1) accumulates in apoplastenriched extracts of the model plant Arabidopsis thaliana in an EDS1-dependent manner ${ }^{5}$. LLP1 is essential for SAR, primarily functioning in the systemic tissue in SAR signal perception or propagation ${ }^{5,6}$. LLP2 (At3g16530), which shares 66\% similarity at the amino acid (AA) level with LLP1, was identified as a possible SARassociated protein along with LLP1 ${ }^{5}$. LLP1 and LLP2 respectively share $61 \%$ and 87\% AA similarity with LLP3 (At3g15356; LECTIN in ${ }^{7}$ ). LLP2 and LLP3 are induced at the transcriptional level by chitin and jasmonic acid (JA), respectively ${ }^{7,8}$, but their physiological roles remain unknown.

For a functional SAR response to occur, two interconnected signalling pathways are required ${ }^{9,10}$. The first of these pathways is primarily associated with $S A^{4,11}$. The second involves pipecolic acid (Pip), and its presumed bioactive derivative $N$ hydroxy-pipecolic acid (NHP) ${ }^{12-14}$. LLP1 has a key role in the latter cascade acting downstream of Pip and upstream of SAR-associated volatile signals to propagate SAR-associated immunity in systemic tissues ${ }^{6}$.

When considering plants in complex natural systems, biotrophic defence signalling cascades associated with SA interact with other stress response pathways. These include abiotic stress responses associated with abscisic acid (ABA) and defence against necrotrophic pathogens and insects controlled by $\mathrm{JA}^{15-17}$. In order to fine tune defence, and optimise resource allocation, there is general antagonism between the three pathways ${ }^{18}$. Studies in Arabidopsis, for example, showed that after SA defences were activated by a hemibiotrophic pathogen, plants also became more susceptible to a necrotrophic pathogen, Alternaria brassicicola, pointing towards a down-regulation in JA-mediated defences ${ }^{19,20}$. Interestingly, the same studies found that this antagonism was restricted to the infected tissues and did not spread systemically during SAR. Recent evidence further convolutes the role of JA in SAmediated defence which appears to be highly dependent on concentration, spatial distribution, and circadian rhythm ${ }^{21-23}$.

It is common for plants to be challenged by abiotic factors at the same time that they are under pathogen attack. Indeed, as climates shift, not only are traditional crops placed under greater stress from factors such as drought and salinity, but also from 
1 emerging infectious diseases and existing pathogens that have expanded their

2 geographical range 24,25 . This threat to crop security increases the necessity for knowledge of interactions between the different stress pathways. Here we show that LLP1 and LLP3 have novel functions in multiple stress responses, and harbor significant potential for engineering multi-stress tolerance in plants.

\section{Results}

\section{LLP3 is essential for local SAR signal generation}

SA is a key component for plant defence against biotrophic pathogens, in both local and systemic tissues. Here, both Col-0 wild type and eds1-2 knockout plants were spray-treated with $1 \mathrm{mM}$ SA. After $24 \mathrm{~h}$, the levels of LLP1 transcripts were induced, and this effect was not dependent on EDS1 (Fig. $1 A_{;}^{5}$ ). In contrast to Lyou et al. ${ }^{7}$, who detected a slight reduction in LLP3 transcript abundance after treatment of plants with $50 \mu \mathrm{M}$ SA, we did not observe a reproducible down regulation of LLP3. Similarly, LLP2 transcript levels did not significantly change in response to SA in either genotype. Similar results were seen if plants were treated with the SA analogue 1,2,3-benzothiadiazole-7-carbothioic acid S-methyl ester (BTH; Supplementary Fig. 1). Thus, LLP1 transcript accumulation, and not that of its homologues $L L P 2$ and $L L P 3$, is regulated by $S A$, and this regulation is independent of EDS1.

Reduced transcript accumulation of $L L P 1, L L P 2$, and $L L P 3$ in RNAi:LLP1-3 plants compromises the ability of the plants to generate or transmit phloem-mobile SAR signal $(s)^{6}$. Because we have so far been unable to generate viable I/p2 mutant plants, we focused on $L L P 3$, whose transcript accumulation was reduced in an IIp3 T-DNA mutant (Supplementary Fig. 2). To analyze SAR, these plants were initially infiltrated in two leaves with either Pst AvrRpm1 or a $10 \mathrm{mM} \mathrm{MgCl}_{2}$ mock control solution. Three days later, two leaves distal to the initial infection were infiltrated with virulent Pst. After another four days, the resulting in planta Pst titres were determined. In wildtype plants, a local Pst AvrRpm1 infection reduced Pst growth in the systemic tissues compared to that in mock-treated plants, indicating the establishment of SAR (Fig. 1B). SAR was fully abolished in Ilp3 mutant plants (Fig. 1B). Ectopic expression of a wildtype copy of $L L P 3$ driven by its native promoter in the Ilp3 mutant background (IIp3-LLP3:LLP3) raised LLP3 transcript accumulation to intermediate 
1 levels between that of wildtype and I/p3 mutant plants (Supplementary Fig. 2). This

2 complemented the SAR-defective phenotype of the Ilp3 mutant (Fig. 1B), ascribing

3 LLP3 an essential role in SAR.

We next tested if LLP3 acts locally or systemically in SAR by using petiole exudates (PEX) from Pst AvrRpm1-inoculated and mock-treated plants. 24 Hours (h) after infiltration of these PEX in recipient plants, the treated leaves were inoculated with Pst and the resulting Pst titers monitored at 4 days post-inoculation (dpi). PEX from infected wildtype plants reduced Pst growth in wildtype recipient plants as compared to PEX from mock-treated wildtype plants (Fig. 1C). Similarly, I/p3 recipient plants responded with reduced Pst growth to PEX from infected wildtype plants, suggesting that $L L P 3$ is not involved in systemic recognition or propagation of SAR signal(s). In contrast, PEX from infected I/p3 donor plants did not reduce Pst growth in wildtype recipient plants (Fig. 1C), suggesting that $L L P 3$ is necessary for local SAR signal generation or transmission. This spatially separates the role in SAR of $L L P 3$ from that of its sequence-related homolog $L L P 1$, which acts systemically in $\mathrm{SAR}^{6}$.

\section{LLP1-3 influence responses to abiotic stress}

Because $L L P 3$ did not show a significant response to SA treatment, but nevertheless influenced SAR, we questioned if LLP3 might be regulated by phytohormones other than SA. Yasuda et al. ${ }^{26}$ showed that $A B A$ and ABA-dependent responses to salinity stress compromised SA signalling and potentially SAR. In order to investigate whether $\mathrm{ABA}$ had an impact upon the transcript levels of $L L P 1, L L P 2$, and LLP3, plants were spray-treated with $100 \mu \mathrm{M}$ ABA and tissues were harvested $24 \mathrm{~h}$ later. In both Col-0 wild type and eds1-2 plants, LLP1 transcript levels were significantly downregulated, while there was no change in transcript levels of either $L L P 2$ or $L L P 3$ (Figure 2A). Thus, ABA downregulates $L L P 1$ transcript accumulation independently of EDS1.

ABA is an important phytohormone in abiotic stress signalling. Therefore, it seemed possible that if $L L P 1$ was transcriptionally regulated by $A B A, \| / p 1-1$ mutant and RNAi:LLP1-3 plants may show an altered phenotype under abiotic stress. To test for aberrant reactions to high salinity, seedlings were germinated and after 6 days transferred to treatment plates with $100 \mathrm{mM} \mathrm{NaCl}$. The length of the primary roots was measured at 6 and 12 days post transfer and normalised to those on control plates 
1 (to which seedlings had also undergone transfer). While Ilp1-1 plants had marginally

2 longer roots than wildtype on control plates (Supplementary Fig. 3), the plants of all

3 genotypes showed a significant reduction in root length when grown on salt compared to control conditions. Notably, both IIp1-1 and RNAi:LLP1-3 plants showed more pronounced salt-induced root growth inhibition as compared to wildtype plants, and RNAi:llp1-3 was significantly more affected than Ilp1-1 (Fig. 2B). To test for a possible contribution of LLP3 to salt-induced root shortening, we transferred I/p3 seedlings and those of two IIp3-LLP3:LLP3 complementation lines to treatment plates with $100 \mathrm{mM} \mathrm{NaCl}$. Similar to Ilp1-1, I/p3 mutants displayed exaggerated root growth inhibition under salt stress, and this phenotype was complemented by ectopic expression of LLP3:LLP3 (Fig. 2C). This might be associated with changes in ABA responses, because ABA-induced root shortening was also exaggerated in the I/p3 mutant, but not in I/p3-LLP3:LLP3 complementation lines (Fig. 2D). However, ABA-induced root shortening was only moderately changed in Ilp1-1 and not changed in RNAi:LLP1-3 plants compared to wild type (Supplementary Fig. 4A). Also, ABA-induced transcript accumulation of the $A B A$ marker gene $R A B 18$ was the same in all genotypes (Supplementary Fig. 4B/C). Therefore, a contribution of ABA to $L L P$-associated root shortening is probably minor. The absence of ABA-associated phenotypes was further supported by the response of RNAi:LLP1-3 plants to progressive drought. There was no physiologically relevant or significant difference between RNAi:LLP1-3 and wild type plants when water consumption and water use efficiency (WUE) under progressive drought, were examined (Supplementary Fig. 5). We therefore posit that the increased sensitivity of the RNAi:LLP1-3 lines to high salinity is most likely mechanistically independent of ABA.

\section{$L L P 3$ responds to MeJA and affects JA-mediated responses}

Another candidate pathway that has been shown to be involved in both biotic defence and salt stress tolerance is the JA pathway ${ }^{27}$. Also, MeJA treatment has been associated with increased $L L P 3$ transcript levels ${ }^{7}$. Here, spray treatment of Arabidopsis with $100 \mu \mathrm{M}$ MeJA increased accumulation of LLP3 transcripts in both Col-0 and eds1-2 plants, suggesting that MeJA induces LLP3 in an EDS1independent manner (Fig. 3A). The accumulation of LLP1 and LLP2 transcripts was 
1 not significantly changed by MeJA. We next investigated whether the reduction of

2 LLP3 in RNAi:LLP1-3 plants would affect JA-mediated defence against a necrotrophic pathogen. Indeed, lesions induced by $A$. brassicicola, a necrotrophic fungus, were larger in RNAi:LLP1-3 plants than in wildtype plants and similar phenotypes were observed in I/p1-1 and I/p3 mutant plants (Fig. 3B/C). This shows that LLP1, LLP2, and/or LLP3 promote defence against necrotrophic pathogens and thus potentially normal JA signalling under biotic stress.

We subsequently tested if compromised JA signalling could have been responsible for the root growth inhibition phenotype of the RNAi:LLP1-3 seedlings on salt. To this end, we again used the root growth inhibition assay, but this time the treatment plates were supplemented with $40 \mu \mathrm{M}$ MeJA. This treatment induced similar results as treatment with $\mathrm{NaCl}$. Both the I/p1-1 mutant and RNAi:LLP1-3 seedlings showed significantly enhanced root length inhibition compared to wildtype (Fig. 3D). Similarly, Ilp3 mutant seedlings displayed exaggerated root growth inhibition on MeJA and this phenotype was complemented by ectopic expression of LLP3:LLP3 (Fig. 3E). JA downstream signalling pathways in the seedlings after 12 days on MeJAsupplemented plates were also aberrant in the RNAi:LLP1-3 seedlings. Transcript accumulation of the JA marker gene PDF1.2 was increased 12 days after transfer of wildtype, Ilp1-1, and Ilp3 seedlings from control to MeJA plates (Fig. 3F and Supplementary Fig. 6A). By contrast, the induction of PDF1.2 transcript accumulation was compromised in RNAi:LLP1-3 plants (Fig. 3F), while the transcript accumulation of VSP2 remained unchanged (Supplementary Fig. 6B).

To determine whether the loss of LLP1-3 was affecting gene expression through changes in hormone biosynthesis, or through downstream interactions, the net content of SA, JA and ABA was measured in RNAi:LLP1-3 plants after treatment with salt. Although there was an increase in net JA and ABA content after salt treatment, there was no significant difference between wildtype and RNAi:LLP1-3 plants (Supplementary Fig. 7). This indicates that signalling aberration does not occur in the biosynthesis of these phytohormones, and that any crosstalk occurs in the pathways downstream of phytohormone biosynthesis. Thus, the three LLP proteins appear to simultaneously promote JA-associated defence against necrotrophic A. brassicicola and JA-associated salt tolerance in a process that is occurring downstream of JA accumulation. 
2 Crosstalk between JA and SA signalling pathways is misregulated in RNAi:LLP1-3 3 plants

4 From the above experiments, the RNAi:LLP1-3 plants show a different level of JA marker gene transcript accumulation under abiotic stress. The SA and JA signalling pathways have multiple points of interaction, normally resulting in antagonistic cross talk $^{18}$. We therefore investigated $P R 1$ transcript accumulation as a marker of SA signalling after watering of mature plants with salt. Whereas PR1 transcript levels were reduced in Col-0 wild type after salt treatment when compared to a mock control, possibly due to the antagonistic relationship between SA and either ABA or MeJA, $P R 1$ transcript levels remained unchanged in $I / p 1-1$ and were upregulated by $\sim 80$-fold in salt- compared to mock-treated RNAi:LLP1-3 plants (Fig. 4A). Hence, LLP1, LLP2, and/or LLP3 might co-operate in compromising responses to salt or associated JA-SA crosstalk events resulting in enhanced SA-associated responses in $R N A i: L L P 1-3$ plants in response to salinity stress.

As MeJA-associated stress was able to induce SA-dependent gene expression in LLP1-3-compromised plants, we investigated whether a local application of MeJA would be sufficient to reconstitute a systemic defence response in the same lines. Using a similar experimental setup to a classical SAR assay, two lower leaves were infiltrated with $100 \mathrm{uM} \mathrm{MeJA}$, and systemic leaves were infiltrated with virulent Pst three days later. The bacterial titres in the systemic leaves at 4 dpi indicated that MeJA, while not affecting bacterial titres in wildtype, was able to reconstitute a SAR response in I/p1-1 mutant plants, but not in Ilp3 or RNAi:LLP1-3 lines (Fig. 4B).

\section{Discussion}

In this paper we show that LLP3 acts locally in SAR signal generation. LLP3 expression is induced by MeJA, and I/p3 mutant plants display JA-associated biotic and abiotic stress tolerance phenotypes (Fig. 3). This implies that local JA responses contribute to SAR signal generation or transmission. Until now, a potential role of JA in SAR has been under debate ${ }^{28,29}$, and JA has been believed to be subject to antagonistic control in local infected tissues undergoing $\mathrm{ETI}^{19,20}$. However, during RPS2-mediated ETI the accumulation of SA and downstream signalling through the NPR3 and NPR4 receptors initiates de novo JA synthesis ${ }^{30}$, and the SA sector in 
1 Arabidopsis immune networks activated during PTI is dependent upon the JA 2 sector $^{31}$. Given that PTI and ETI have some convergent signalling pathways, 3 including through SA accumulation ${ }^{32,33}$, it is likely that JA may have a more important

SA and Pip are thought to function via interconnected signalling pathways during $S A R^{9,10}$. LLP1 transcript accumulation is increased in response to $S A$ (Fig. $1 \mathrm{~A}$ ), but is dispensable for SA-induced immunity ${ }^{5}$. LLP1 further promotes systemic SAR signal recognition or propagation downstream of Pip and drives a positive feedback loop propagating volatile monoterpene emissions as airborne SAR cues ${ }^{6}$. Notably, MeJAinduced root growth inhibition was only marginally, if at all, exaggerated in Pipdeficient ald $1^{34}$ plants and in different monoterpene emission-compromised ${ }^{6}$ mutants (Supplementary Fig. 8). Therefore, LLP1 might interact with an LLP3-associated SAR signalling component in a pathway that is mostly separate from its role in Pip signalling and monoterpene transmission.

JA activates two separate signalling pathways, depending upon which other signals/factors are detected at the same time. This allows the plant to use JA to finetune responses to multiple stresses ${ }^{35}$. The exaggerated root shortening and enhanced $A$. brassicicola susceptibility phenotypes of the $\|p 1-1\| p$,3 , and RNAi:LLP1-3 plants suggest that there is a misregulation in signalling at a point upstream of one of JA's two key pathway regulators, MYC2 and ERF1 ${ }^{36}$. Transcript levels of the ERF1 pathway marker gene PDF1.2 were reduced in MeJA-treated RNAi:LLP1-3 plants, whereas the MYC2 pathway marker gene VSP2 was not misregulated in the same plants (Fig. 3 and Supplementary Fig. 6). ERF1 is a key transcription factor activated in conjunction with ethylene signalling, which is implicated in defence against necrotrophic pathogens ${ }^{37,38}$, and is strongly induced in response to salt stress ${ }^{39}$. The susceptibility of $\| / p 1-1$, Ilp3, and RNAi:LLP1-3 plants to the pathogen $A$. brassisicola thus further supports a misregulation of the ERF1regulated branch of JA signalling in these mutants. Together, the data suggest that LLP1, 2, and/or 3 influence JA responses through ERF1 (Fig. 4C). 
1 Although observed physiologically in all genotypes tested, misregulated SA-JA cross

2 talk events were observed at the molecular level, i.e. PDF1.2 and PR1 transcript accumulation changes, only in RNAi:LLP1-3 plants. This hints at possible additive roles of $L L P 1, L L P 2$, and $L L P 3$ in this process. During SAR, however, the roles of $L L P 1$ and $L L P 3$ appear to be spatially separated with $L L P 1$ acting systemically and LLP3 promoting local SAR signal generation. This might explain why MeJA enhanced the systemic resistance of Ilp1-1, but not RNAi:LLP1-3 plants against Pst if $L L P 3$ is required locally to drive JA-associated SAR signal generation or transmission through ERF1 (Fig. 4C). During SAR, SA-JA antagonism is observed locally, but not in the systemic tissues ${ }^{19,20}$. Perhaps, LLP1 fine-tunes incoming signals to avoid antagonistic trade-offs between SA- and JA-mediated defences in the systemic tissue during SAR (Fig. 4C).

The recently suggested spatial role of JA signalling in the perimeter of SA-induced HR lesions ${ }^{21}$ might explain how JA locally influences SAR signal generation. High SA levels in the core of the lesion promote LLP1 transcript accumulation, while JA accumulation in the rim of the lesion drives up $L L P 3$ expression. We hypothesize that this signal is then relayed through the ERF1 pathway affecting salt tolerance, defence against necrotrophic pathogens, and also driving SAR signal emission from this site (Fig. 4C). The role of LLP1 allows this pathway to act synergistically with both the SA- and Pip-dependent systemic defence signals, creating an interwoven network necessary for SAR-associated defence priming. Priming of SA defences in the absence of deleterious effects on JA defences further assigns a high potential to LLP-associated SAR signalling components for application in future durable plant protection strategies. A possible exploitation of LLP-associated signaling moieties towards resource-efficient defence priming will be subject of further study.

\section{Methods}

Plant materials and growth conditions

A. thaliana ecotype Columbia-0 (Col-0) was used as the wild type control throughout all experiments. Transgenic lines IIp1-1, eds1-2, ald1, ggpps12, tps24-1, tps24-2, and RNAi:LLP1-3 have been described previously ${ }^{5,6,34,40,41}$. RNAi_LLP1-3 line C3 13-16 was used for all experiments. SALK_030762 with a T-DNA insertion in LLP3 
1 (At3G15356) was obtained from the Nottingham Arabidopsis Stock Center ${ }^{42}$, and 2 propagated to homozygosity. Plants that were homozygous for the T-DNA insertion were used for all experiments and as the parental line for generating IIp3-LLP3:LLP3 complementation lines 3.02 and 4.01. For the latter, LLP3:LLP3 constructs were generated from Col-0 wild type genomic DNA. The native promoter was chosen from $\sim 2$ kilo base pairs upstream to the LLP3 transcriptional start site, and the LLP3:LLP3 target sequence was isolated by PCR using the primers LLP3:LLP3-F and LLP3:LLP3-R (Supplementary Table 1). The resulting DNA fragment was cloned into

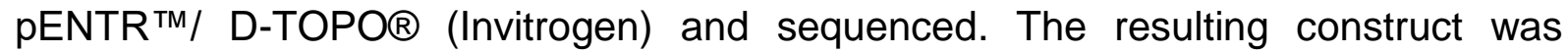
transferred to the binary Gateway® cloning vector pBGWFS7, $0^{43}$, with the GUS sequence removed using the restriction enzyme Nrul (pBGWFS7,0 $\Delta G U S$ ). The resulting binary vector was transformed into Agrobacterium tumefaciens strain GV3101 and used for plant transformation by floral dip ${ }^{44}$. Transgenic T1 plants were selected via 200g/L BASTA spray (Hoechst, Germany). Experiments were performed in T3 plants. LLP3 transcript levels were determined by RT-qPCR as described below with the LLP3 primer sets c1 or c2 (Supplementary Table 1).

Plants were grown on potting soil (without fertilizer) mixed with sand in 5:1 ratio, and kept under short day conditions (10 hours (h) light with an intensity of $100 \mu \mathrm{E} \mathrm{m} \mathrm{m}^{-2}$ at $22^{\circ} \mathrm{C}$ and $14 \mathrm{~h}$ dark at $18^{\circ} \mathrm{C}, 70 \%$ relative humidity).

\section{Phytohormone treatments}

To analyse $L L P 1-3$ transcript accumulation in response to phytohormone treatment, green tissues of 2- to 3-week old plants were sprayed until drop-off with $1 \mathrm{mM} \mathrm{SA}$ (Sigma Aldrich), $100 \mu \mathrm{M}$ MeJA (Sigma Aldrich), or $100 \mu \mathrm{M}$ ABA (Sigma Aldrich) dissolved in $0.1 \% \mathrm{MgCl}_{2}, 0.01 \%$ Tween $^{\circ} 20$, and $0.025 \% \mathrm{MeOH}$. Plants of the same age were sprayed with $0.1 \% \mathrm{MgCl}_{2}, 0.01 \%$ Tween $^{\circ} 20,0.025 \% \mathrm{MeOH}$ as the mock control treatment. Leaf samples were taken at 8 and $24 \mathrm{~h}$ after treatment and flash frozen in liquid $\mathrm{N}_{2}$.

\section{Pathogen infection assays}

Pseudomonas syringae pathovar tomato (Pst) and Pst AvrRpm1 were maintained as described $^{5}$. To induce a SAR response, plants were infiltrated in their first two true leaves with $1 \times 10^{6} \mathrm{CFU} / \mathrm{mL}$ of Pst/AvrRPM1. Three days later, two systemic leaves 
1 were infiltrated with $1 \times 10^{5} \mathrm{CFU} / \mathrm{mL}$ of Pst. The resulting in planta bacterial titres were

2 determined at $4 \mathrm{dpi}$ as described ${ }^{6}$. A. brassicicola was maintained on malt medium

3 (3\% malt extract (Merck), 1.5\% agar-agar (Roth)) and transferred to oat plates (oats

4 (Alnatura) in 1.5\% w/v agar-agar) before experiments. Mycelium was solved in MKP

5 buffer $\left(62 \mathrm{mM} \mathrm{KH}_{2} \mathrm{PO}_{4}, 0.01 \%\right.$ glucose, $0.01 \%$ Tween $\left.^{\circledR} 20\right)$ until a concentration of

6200 spores/ $\mu \mathrm{L}$ was achieved. Plants were inoculated by placing $3 \mu \mathrm{L}$ droplets onto

7 the third and fourth true leaf. The resulting lesion sizes were determined at $5 \mathrm{dpi}$

8 using ImageJ. Cell death was visualized using trypan blue staining as described ${ }^{45}$.

9

\section{Petiole exudate experiments}

Petiole exudate experiments were performed as described ${ }^{6}$. In short, Pst AvrRpm1inoculated leaves were cut in the middle of the rosette at $24 \mathrm{~h}$ post-inoculation, and incubated with their petioles in $1 \mathrm{mM}$ EDTA. After $1 \mathrm{~h} 6$ leaves per exudate were transferred to $2.0 \mathrm{~mL}$ of sterilized water and allowed to exude for $48 \mathrm{~h}$. The resulting PEX solutions were filter-sterilized (Millipore, $0.22 \mu \mathrm{m}$ ) and supplemented with $\mathrm{MgCl}_{2}$ to a final concentration of $1 \mathrm{mM} .24 \mathrm{~h}$ after syringe infiltration of the PEX in leaves of naïve recipient plants, the infiltrated leaves were inoculated with $10^{5} \mathrm{cfu} \mathrm{mL}^{-1}$ of $P s t$, in planta titres of which were determined at $4 \mathrm{dpi}$ as described above.

\section{Root length inhibition assays}

For root growth inhibition measurements, seedlings were sterilised in $75 \%$ followed by $100 \% \mathrm{EtOH}$ (Merck), dried, and sown on 1x Murashige Skoog medium including vitamins (Duchefa) with $0.1 \%$ cefotaxim (Acros Organics) and $0.25 \%$ Carbenicillin (Roth). Seedlings were transferred after 6 days to treatment plates containing either $10 \mu \mathrm{M} \mathrm{ABA}, 100 \mathrm{mM} \mathrm{NaCl}$, or $40 \mu \mathrm{M}$ MeJA (Sigma-Aldrich), or to control MS plates. All plates were placed upright in the growth chamber under long day conditions, and the seedlings were photographed 6 and 12 days post-transfer. Root length was measured using ImageJ. The seedlings were harvested, pooled per genotype and treatment, and flash frozen in $\mathrm{N}_{2}$ for RNA extraction.

\section{Phytohormone content measurements}

$A B A$ in seedlings was measured as described ${ }^{46}$. In short, the frozen material was

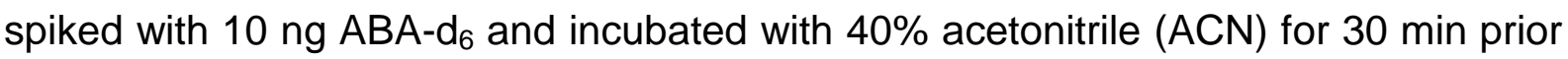


1 acidification with phosphoric acid and extraction with tert-butyl methyl ether. The

2 organic extract was passed over a Chromabond NH2 500 mg solid phase extraction

3 column (Macherey-Nagel, Düren, Germany). The eluate was diluted with distilled water and passed over a Chromabond C18ec $100 \mathrm{mg}$ solid phase extraction column (Macherey-Nagel). The eluate was evaporated in a vacuum concentrator, dissolved and fractionated by RP-HPLC using a Nucleodur 100-5 C18ec 125x4.6 mm column (Macherey-Nagel). The ABA-containing fraction was collected, evaporated to dryness and methylated with $2 \mathrm{M}$ (trimethylsilyl)diazomethane $/$ methanol $=1: 19$. After evaporation, the residue was dissolved in $A C N$ and analysed by gas chromatography-mass spectrometry using a VF-5ms column (Agilent, St. Louis, MO, USA) and helium as carrier gas at a flow rate of $1.5 \mathrm{ml} / \mathrm{min}$. The ions with $\mathrm{m} / \mathrm{z} 190$ $(A B A)$ and $194\left(A B A-d_{6}\right)$ were used for quantification and the ions at 134 and 162 $(A B A)$ and 138 and $166\left(A B A-d_{6}\right)$ were used as qualifiers.

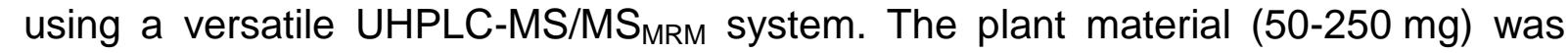
placed in $2 \mathrm{~mL}$ bead beater tubes (CKMix-2 mL, Bertin Technologies, Montigny-le-Bretonneux, France). An aliquot of the internal standard $(20 \mu \mathrm{L})$, containing ABA-d 6 (2.5 $\mu \mathrm{g} / \mathrm{mL}), \quad S A-d_{4}(2.5 \mu \mathrm{g} / \mathrm{mL})$, and $J A-d_{5} \quad(25 \mu \mathrm{g} / \mathrm{mL})$ in acetonitrile was added to the plants and incubated for $30 \mathrm{~min}$ at room temperature. After extractive grinding with ethyl acetate $(1 \mathrm{~mL})$ in a bead beater (Precellys Homogenizer, Bertin Technologies, Montigny-le-Bretonneux, France) the supernatant was membrane filtered $(0.45 \mu \mathrm{m})$, evaporated to dryness, resolved in acetonitrile $(70 \mu \mathrm{L})$ and injected into the LC-MS/MS-system $(2 \mu \mathrm{L})$.

For LC-MS/MS analysis a QTRAP $6500^{+}$mass spectrometer (Sciex, Darmstadt, Germany) was used to acquire electrospray ionization (ESI) mass spectra and product ion spectra. Negative and positive ions were detected in the scheduled multiple reaction monitoring (MRM) mode.

For analysis of ABA, SA and JA, the MS/MS parameters were tuned to achieve fragmentation of the $[\mathrm{M}-\mathrm{H}]^{-}$and $[\mathrm{M}+\mathrm{H}]^{+}$molecular ions into specific product ions to receive a qualifier and a quantifier transition for every compound.

Chromatography was performed by means of an ExionLC UHPLC system (Shimadzu Europa $\mathrm{GmbH}$, Duisburg, Germany) equipped with a Kinetex F5 column $(100 \times 2.1 \mathrm{~mm}, 100 \AA ̊, 1.7 \mu \mathrm{m}$, Phenomenex, Aschaffenburg, Germany). Operated 
1 with a flow rate of $0.4 \mathrm{~mL} / \mathrm{min}$ using $0.1 \%$ formic acid in water $(\mathrm{v} / \mathrm{v})$ as solvent $\mathrm{A}$ and

$2 \quad 0.1 \%$ formic acid in acetonitrile $(\mathrm{v} / \mathrm{v})$ as solvent $\mathrm{B}$, chromatography was performed 3 with the following gradient: $0 \% \mathrm{~B}$ held for $2 \mathrm{~min}$, increased in $1 \mathrm{~min}$ to $30 \% \mathrm{~B}$, in $412 \mathrm{~min}$ to $30 \% \mathrm{~B}$, increased in $0.5 \mathrm{~min}$ to $100 \% \mathrm{~B}$, held $2 \mathrm{~min}$ isocratically at $100 \% \mathrm{~B}$, decreased in $0.5 \mathrm{~min}$ to $0 \% \mathrm{~B}$, held $3 \mathrm{~min}$ at $0 \% \mathrm{~B}$. Data acquisition and instrumental control were performed using Analyst 1.6.3 software (Sciex, Darmstadt, Germany).

\section{Salt pouring experiments}

Plants were watered with distilled water or $300 \mathrm{mM} \mathrm{NaCl}$ three times with four day intervals starting from 4 weeks after germination. Leaf tissue was harvested 4 days after the final salt treatment, weighed, and flash frozen in liquid $\mathrm{N}_{2}$.

\section{$R N A$ isolation and $R T-q P C R$}

Total RNA was extracted from leaves and seedlings using TriReagent (SigmaAldrich) following the manufacturer's instructions. cDNA was synthesized using SuperScriptll reverse transcriptase (Invitrogen). Real-time quantitative PCR was performed using the Sensimix SYBR low-rox kit (Bioline) and the primers in Supplementary Table 2, with UBIQUITIN as the reference gene. Endogenous LLP3 transcript accumulation was determined with the primers LLP3-F and LLP3-R. qPCR was performed on a 7500 real-time PCR system (Applied Biosystems). Transcript accumulation was analysed using the 7500 Fast System Software 1.3.1.

\section{Drought assay}

The progressive drought experiment was performed as described ${ }^{47}$. In brief, Arabidopsis plantlets were exposed to a slowly increasing water deficit by minimizing evaporation and withholding watering under short day conditions ( $8 \mathrm{~h}$ light). Water consumption per plant was recorded from 18 to 73 days after seeding. Above-ground material was used for determining the dry-weight biomass and WUE was expressed as the ratio of biomass to consumed water.

\section{Statistics}

Data was analysed in GraphPad Prism 8 for Windows. If necessary, outliers were removed using a Grubbs' test $(\alpha=0.05)$. Normal distribution of the data was checked 
1 using D'Agostino Pearson ( $\alpha=0.01$ ). Data that showed normal distribution was tested

2 for significance using an unpaired one-way ANOVA with Tukey's multiple comparison test, and data that was not normally distributed was tested using a Kruskal-Wallis test with a Dunn's multiple comparison test.

\section{Supplementary Material}

7 Supplementary Table 1 Primers for LLP3:LLP3 construct generation and qPCR

8 Supplementary Table 2 Primers for qPCR

9 Supplementary Figure $1 \mathrm{BTH}$ induces transcript accumulation of LLP1.

Supplementary Figure 2 LLP3 transcript levels in I/p3 and Ilp3-LLP3:LLP3 complementation lines

Supplementary Figure $3 L L P 1$ moderately influences primary root growth.

Supplementary Figure $4 \mathrm{llp} 1-1$ and $R N A i: L L P 1-3$ lines do not show an altered response to $A B A$

15 Supplementary Figure $5 L L P 1-3$ do not affect the response to drought stress.

Supplementary Figure $6 \mathrm{JA}$-associated marker gene expression

Supplementary Figure 7 LLP1-3 do not influence phytohormone accumulation in response to salt

Supplementary Figure 8 Salt- and MeJA-induced primary root growth inhibition in seedlings of SAR-associated mutant lines

\section{Acknowledgments}

We thank Miriam Lenk for critically reading the manuscript. This work was funded by the Deutsche Forschungsgemeinschaft (DFG) as part of SFB924 (projects A12 (BP), B01 (EG), B12 (CD), and project B06 to ACV). 


\section{References}

1. Boutrot, F. \& Zipfel, C. Function, Discovery, and Exploitation of Plant Pattern Recognition Receptors for Broad-Spectrum Disease Resistance. Annu Rev Phytopathol 55, 257-286 (2017).

2. Jones, J.D. \& Dangl, J.L. The plant immune system. Nature 444, 323-9 (2006).

3. Cui, H., Tsuda, K. \& Parker, J.E. Effector-triggered immunity: from pathogen perception to robust defense. Annu Rev Plant Biol 66, 487-511 (2015).

4. Vlot, A.C., Dempsey, D.A. \& Klessig, D.F. Salicylic Acid, a multifaceted hormone to combat disease. Annu Rev Phytopathol 47, 177-206 (2009).

5. Breitenbach, H.H. et al. Contrasting Roles of the Apoplastic Aspartyl Protease APOPLASTIC, ENHANCED DISEASE SUSCEPTIBILITY1-DEPENDENT1 and LEGUME LECTIN-LIKE PROTEIN1 in Arabidopsis Systemic Acquired Resistance. Plant Physiol 165, 791-809 (2014).

6. Wenig, M. et al. Systemic acquired resistance networks amplify airborne defense cues. Nat Commun 10, 3813 (2019).

7. Lyou, S.H. et al. The Arabidopsis AtLEC gene encoding a lectin-like protein is up-regulated by multiple stimuli including developmental signal, wounding, jasmonate, ethylene, and chitin elicitor. Mol Cells 27, 75-81 (2009).

8. Ramonell, K. et al. Loss-of-function mutations in chitin responsive genes show increased susceptibility to the powdery mildew pathogen Erysiphe cichoracearum. Plant Physiol 138, 1027-36 (2005).

9. Kim, Y., Gilmour, S.J., Chao, L., Park, S. \& Thomashow, M.F. Arabidopsis CAMTA Transcription Factors Regulate Pipecolic Acid Biosynthesis and Priming of Immunity Genes. Mol Plant 13, 157-168 (2020).

10. Sun, T. et al. Redundant CAMTA Transcription Factors Negatively Regulate the Biosynthesis of Salicylic Acid and N-Hydroxypipecolic Acid by Modulating the Expression of SARD1 and CBP60g. Mol Plant 13, 144-156 (2020).

11. Gao, Q.M., Zhu, S., Kachroo, P. \& Kachroo, A. Signal regulators of systemic acquired resistance. Front Plant Sci 6, 228 (2015).

12. Chen, Y.C. et al. N-hydroxy-pipecolic acid is a mobile metabolite that induces systemic disease resistance in Arabidopsis. Proc Natl Acad Sci U S A 115, E4920-E4929 (2018).

13. Hartmann, M. et al. Flavin Monooxygenase-Generated N-Hydroxypipecolic Acid Is a Critical Element of Plant Systemic Immunity. Cell 173, 456-469 e16 (2018).

14. Wang, C. et al. Pipecolic acid confers systemic immunity by regulating free radicals. Sci Adv $\mathbf{4}$, eaar4509 (2018).

15. Ellis, C. \& Turner, J.G. The Arabidopsis mutant cev1 has constitutively active jasmonate and ethylene signal pathways and enhanced resistance to pathogens. Plant Cell 13, 1025-33 (2001).

16. Farmer, E.E. \& Ryan, C.A. Octadecanoid Precursors of Jasmonic Acid Activate the Synthesis of Wound-Inducible Proteinase Inhibitors. Plant Cell 4, 129-134 (1992).

17. Saijo, Y. \& Loo, E.P. Plant immunity in signal integration between biotic and abiotic stress responses. New Phytol 225, 87-104 (2020).

18. Pieterse, C.M., Van der Does, D., Zamioudis, C., Leon-Reyes, A. \& Van Wees, S.C. Hormonal modulation of plant immunity. Annu Rev Cell Dev Biol 28, 489-521 (2012).

19. Spoel, S.H., Johnson, J.S. \& Dong, X. Regulation of tradeoffs between plant defenses against pathogens with different lifestyles. Proc Natl Acad Sci U S A 104, 18842-7 (2007).

20. Wittek, F. et al. Folic acid induces salicylic acid-dependent immunity in Arabidopsis and enhances susceptibility to Alternaria brassicicola. Mol Plant Pathol 16, 616-22 (2015).

21. Betsuyaku, S. et al. Salicylic Acid and Jasmonic Acid Pathways are Activated in Spatially Different Domains Around the Infection Site During Effector-Triggered Immunity in Arabidopsis thaliana. Plant Cell Physiol 59, 8-16 (2018). 
22. Karapetyan, S. \& Dong, X. Redox and the circadian clock in plant immunity: A balancing act. Free Radic Biol Med 119, 56-61 (2018).

23. Mur, L.A., Kenton, P., Atzorn, R., Miersch, O. \& Wasternack, C. The outcomes of concentration-specific interactions between salicylate and jasmonate signaling include synergy, antagonism, and oxidative stress leading to cell death. Plant Physiol 140, 249-62 (2006).

24. Anderson, P.K. et al. Emerging infectious diseases of plants: pathogen pollution, climate change and agrotechnology drivers. Trends Ecol Evol 19, 535-44 (2004).

25. Bebber, D.P. Range-expanding pests and pathogens in a warming world. Annu Rev Phytopathol 53, 335-56 (2015).

26. Yasuda, M. et al. Antagonistic interaction between systemic acquired resistance and the abscisic acid-mediated abiotic stress response in Arabidopsis. Plant Cell 20, 1678-92 (2008).

27. Valenzuela, C.E. et al. Salt stress response triggers activation of the jasmonate signaling pathway leading to inhibition of cell elongation in Arabidopsis primary root. J Exp Bot 67, 4209-20 (2016).

28. Attaran, E., Zeier, T.E., Griebel, T. \& Zeier, J. Methyl salicylate production and jasmonate signaling are not essential for systemic acquired resistance in Arabidopsis. Plant Cell 21, 95471 (2009).

29. Truman, W., Bennett, M.H., Kubigsteltig, I., Turnbull, C. \& Grant, M. Arabidopsis systemic immunity uses conserved defense signaling pathways and is mediated by jasmonates. Proc Natl Acad Sci U S A 104, 1075-80 (2007).

30. Liu, L. et al. Salicylic acid receptors activate jasmonic acid signalling through a non-canonical pathway to promote effector-triggered immunity. Nat Commun 7, 13099 (2016).

31. Hillmer, R.A. et al. The highly buffered Arabidopsis immune signaling network conceals the functions of its components. PLoS Genet 13, e1006639 (2017).

32. Peng, Y., van Wersch, R. \& Zhang, Y. Convergent and Divergent Signaling in PAMP-Triggered Immunity and Effector-Triggered Immunity. Mol Plant Microbe Interact 31, 403-409 (2018).

33. Sun, T. et al. ChIP-seq reveals broad roles of SARD1 and CBP60g in regulating plant immunity. Nat Commun 6, 10159 (2015).

34. Navarova, H., Bernsdorff, F., Doring, A.C. \& Zeier, J. Pipecolic acid, an endogenous mediator of defense amplification and priming, is a critical regulator of inducible plant immunity. Plant Cell 24, 5123-41 (2012).

35. Kazan, K. \& Manners, J.M. JAZ repressors and the orchestration of phytohormone crosstalk. Trends Plant Sci 17, 22-31 (2012).

36. Kazan, K. Diverse roles of jasmonates and ethylene in abiotic stress tolerance. Trends Plant Sci 20, 219-29 (2015).

37. Berrocal-Lobo, M., Molina, A. \& Solano, R. Constitutive expression of ETHYLENE-RESPONSEFACTOR1 in Arabidopsis confers resistance to several necrotrophic fungi. Plant $J$ 29, 23-32 (2002).

38. Lorenzo, O., Piqueras, R., Sanchez-Serrano, J.J. \& Solano, R. ETHYLENE RESPONSE FACTOR1 integrates signals from ethylene and jasmonate pathways in plant defense. Plant Cell 15, 165-78 (2003).

39. Cheng, M.C., Liao, P.M., Kuo, W.W. \& Lin, T.P. The Arabidopsis ETHYLENE RESPONSE FACTOR1 regulates abiotic stress-responsive gene expression by binding to different cisacting elements in response to different stress signals. Plant Physiol 162, 1566-82 (2013).

40. Bartsch, M. et al. Salicylic acid-independent ENHANCED DISEASE SUSCEPTIBILITY1 signaling in Arabidopsis immunity and cell death is regulated by the monooxygenase FMO1 and the Nudix hydrolase NUDT7. Plant Cell 18, 1038-51 (2006).

41. Riedlmeier, M. et al. Monoterpenes Support Systemic Acquired Resistance within and between Plants. Plant Cell 29, 1440-1459 (2017). 
42. Alonso, J.M. et al. Genome-wide insertional mutagenesis of Arabidopsis thaliana. Science 301, 653-7 (2003).

43. Karimi, M., Inze, D. \& Depicker, A. GATEWAY vectors for Agrobacterium-mediated plant transformation. Trends Plant Sci 7, 193-5 (2002).

44. Logemann, E., Birkenbihl, R.P., Ulker, B. \& Somssich, I.E. An improved method for preparing Agrobacterium cells that simplifies the Arabidopsis transformation protocol. Plant Methods 2, 16 (2006).

45. Pogany, M. et al. Dual roles of reactive oxygen species and NADPH oxidase RBOHD in an Arabidopsis-Alternaria pathosystem. Plant Physiol 151, 1459-75 (2009).

46. Avramova, V. et al. Carbon isotope composition, water use efficiency, and drought sensitivity are controlled by a common genomic segment in maize. Theor Appl Genet 132, 53-63 (2019).

47. Yang, Z. et al. Leveraging abscisic acid receptors for efficient water use in Arabidopsis. Proc Natl Acad Sci U S A 113, 6791-6 (2016). 


\section{Figure captions}

Figure 1 LEGUME LECTIN-LIKE PROTEIN3 (LLP3) promotes systemic acquired resistance (SAR) signal generation/transmission. (A) LLP3 transcript accumulation is not affected by salicylic acid (SA). 4-week-old Col-0 wild type and eds1-2 Arabidopsis plants were spray-treated with $1 \mathrm{mM} \mathrm{SA}$, and 24 hours (h) later LLP1, LLP2, and $L L P 3$ transcript accumulation was determined by RT-qPCR. Transcript accumulation was normalized to that of UBIQUITIN and is shown relative to the normalized transcript levels in the appropriate mock controls. Bars represent the $\log _{2}($ mean $) \pm$ SEM of four biologically independent replicates. The letters above the bars indicate statistically significant differences (one-way ANOVA, $n=4, P<0.05, F=4.623, D F=22$ ). (B) $L L P 3$ is required for SAR. Plants were infiltrated locally with either Pst AvrRpm1 (SAR) or with $10 \mathrm{mM} \mathrm{MgCl}_{2}$ as the mock control (M). To monitor SAR, 3 days after the primary treatment leaves distal to the initial treatment site were infiltrated with Pst. Plant lines included IIp3 mutants and 2 independently transformed complementation lines carrying a transgene driving LLP3 expression from its native promoter (I3-L3:L3 3.02 and 4.01). Box plots represent average $P s t$ titres in systemic leaves at 4 days post-inoculation (dpi) from 4 biologically independent experiments, including 3 replicates each $\pm \min$ and $\max$ values. The letters above the box plots indicate statistically significant differences (Kruskal-Wallis test, $P<0.05, \quad n=12, \quad K W$ statistic=101.4). (C) $L L P 3$ is required to send, but not to receive phloem-mobile SAR signals. Leaves of donor plants were inoculated with Pst AvrRpm1 (S) or with the appropriate mock control (M). After $24 \mathrm{~h}$, petiole exudates were collected from the donor plants and infiltrated into leaves of naïve recipient plants. 24h later, the treated leaves were challenged with Pst. Bars represent average Pst titres at $4 \mathrm{dpi}$ from 3 biologically independent experiments, including 3 replicates each \pm SD. The letters above the bars indicate statistically significant differences (one-way ANOVA, $n=9$, $\mathrm{P}<0.05, \mathrm{~F}=6.258, \mathrm{DF}=35)$.

Figure $2 \angle L P 1, L L P 2$, and/or $L L P 3$ compromise Arabidopsis responses to salt stress. (A) LLP1 transcript accumulation is reduced after ABA treatment. Col-0 wild type and

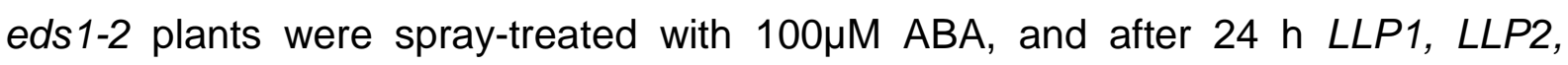
and/or LLP3 transcript accumulation was determined by RT-qPCR. Transcript 
1 accumulation was normalized to that of UBIQUITIN and is shown relative to the

2 normalized transcript levels in the appropriate mock controls. Bars represent the $\log _{2}$ (mean) \pm SEM of three biologically independent replicates. The letters above the bars indicate statistically significant differences (one-way ANOVA, $n=3, P<0.05$, $\mathrm{F}=6.291, \mathrm{DF}=40) .(\mathrm{B} / \mathrm{C}) L L P 1, L L P 2$, and/or $L L P 3$ compromise salt-associated root growth inhibition. Seedlings of Col-0 wild type, Ilp1-1, and RNAi:LLP1-3 (B) and of IIp3 and two IIp3-LLP3:LLP3 complementation lines (I3-L3:L3 3.02 and 4.01; C) were germinated on control MS plates, and after 6 days transferred to either further control plates, or to plates supplemented with $100 \mathrm{mM} \mathrm{NaCl}$. Primary root length was measured at 6 and 12 days post transfer and normalized to that of the same genotype on control plates. Box plots represent average normalized root length \pm min and max values. The letters above the box plots indicate statistically significant differences (B: one-way ANOVA, $\mathrm{P}=<0.05, \mathrm{~F}=30.70$, $\mathrm{DF}=233$, for day 6 , Col-0 $\mathrm{n}=48$, IIp1-1 $\mathrm{n}=38$, RNAi:LLP1 $\mathrm{n}=40$, for day 12 Col-0 $\mathrm{n}=48, \| / p 1-1 \mathrm{n}=29$, RNAi:LLP1-3 $\mathrm{n}=21$; C: Day 6: Kruskal-Wallis test, $\mathrm{P}=<0.05, \mathrm{KW}$ test statistic $=165.5$, Col $-0 \mathrm{n}=83$, IIp3 $n=86$, I3-L3:L3 3.02 n=96, I3-L3:L3 4.01 n=89. Day 12: one-way ANOVA, $\mathrm{P}=<0.05, \mathrm{~F}=25.08, \mathrm{DF}=519$, Col-0 $\mathrm{n}=81$, I/p3 $\mathrm{n}=84$, I3-L3:L3 $3.02 \mathrm{n}=94$, I3-L3:L3 4.01 $\mathrm{n}=84)$. These experiments were repeated $3(\mathrm{C})$ to $4-8$ times $(B)$ with comparable results. (D) LLP3 compromises root growth inhibition on $10 \mu \mathrm{M} A B A$. Col-0 wild type, IIp3 and two IIp3-LLP3:LLP3 complementation lines were treated as described in $(\mathrm{B} / \mathrm{C})$, and the treatment plates were supplemented with $10 \mu \mathrm{M} A B A$. Box plots represent average normalized root length $\pm \min$ and max values. The letters above the box plots indicate statistically significant differences (Day 6: one-way ANOVA, $\mathrm{P}=<0.05, \mathrm{~F}=76.10$, DF=538, Col-0 $\mathrm{n}=76$, Ilp3 $\mathrm{n}=86,3.01 \mathrm{n}=89$, I3-L3:L3 $3.02 \mathrm{n}=98$, 13-L3:L3 4.01 $\mathrm{n}=94,8.01 \mathrm{n}=96$. Day 12: Kruskal-Wallis test, $P=<0.05, \mathrm{KW}$ test statistic $=121.1$, Col-0 $n=85$, IIp3 $n=90$, /3-L3:L3 3.02 $n=100$, I3-L3:L3 4.01 n=97). This experiment was repeated 3 times with comparable results.

Figure $3 L L P 1, L L P 2$, and/or $L L P 3$ differentially affect jasmonic acid (JA)-associated responses in Arabidopsis. (A) LLP3 transcript accumulation is induced by methyl jasmonate (MeJA). Col-0 wild type and eds1-2 plants were spray-treated with $100 \mu \mathrm{M}$ MeJA, and after $24 \mathrm{~h}$ LLP1, LLP2, and/or LLP3 transcript accumulation was determined by RT-qPCR. Transcript accumulation was normalized to that of 
1 UBIQUITIN and is shown relative to the normalized transcript levels in the

2 appropriate mock controls. Bars represent the $\log _{2}$ (mean) \pm SEM of four biologically

3 independent replicates. The letters above the bars indicate statistically significant differences (one-way ANOVA, $\mathrm{n}=4, \mathrm{P}<0.05, \mathrm{~F}=4.493, \mathrm{DF}=45)$. (B/C) $L L P 1, L L P 2$, and/or LLP3 promote JA-associated defence against Alternaria brassicicola. Droplets containing spores of the necrotrophic fungus $A$. brassicicola were placed on the leaves of four-week-old Col-0 wild type, IIp1-1, IIp3, and RNAi:LLP1-3 plants. Resulting lesions were photographed $(B)$ and measured $(C) 5$ days later. Box plots in (C) represent mean lesion diameters from 4 biologically independent experiments including 15 replicates each \pm min and max values. The letters above the box plots indicate statistically significant differences (Kruskal-Wallis test, $\mathrm{P}=<0.05, \mathrm{KW}$ test statistic $=24.10, \mathrm{n}=60$ for all genotypes). (D/E) LLP1, LLP2, and/or LLP3 compromise JA-associated root growth inhibition. Seedlings of Col-0 wild type, IIp1-1, and RNAi:LLP1-3 (D) and of IIp3 and two IIp3-LLP3:LLP3 complementation lines (I3-L3:L3 3.02 and 4.01 ; E) were germinated on control MS plates, and after 6 days transferred to either further control plates, or to plates supplemented with $40 \mu \mathrm{M}$ MeJA. Primary root length was measured at 6 and 12 days post transfer and normalized to that of the same genotype on control plates. Box plots represent average normalized root length \pm min and max values. The letters above the box plots indicate statistically significant differences (D: Day 6: one-way ANOVA, $F=44.87, D F=147$, Col-0 $n=29$, IIp1-1, RNAi:LLP1-3 n=30. Day 12: one-way ANOVA, F=74.62, DF=175, Col-0 n=29, IIp1-1 $\mathrm{n}=30, R N A i: L L P 1-3 \mathrm{n}=28 ; \mathrm{E}$ : Day 6: one-way ANOVA, $\mathrm{P}<0.05, \mathrm{~F}=61.40$, $D F=541$, Col-0 n=71, IIp3 $n=85$, I3-L3:L3 $3.02 n=94$, I3-L3:L3 $4.01 n=97$. Day 12: Kruskal-Wallis test, $P=<0.05$, KW test statistic $=140.7$, Col-0 $n=63$, I/p3 $n=73$, /3L3:L3 3.02 $n=94$, I3-L3:L3 $4.01 n=97,8.01 n=95)$. These experiments were repeated 3 (E) to 5 times (D) with comparable results. (F) LLP1, LLP2, and/or LLP3 compromise MeJA-induced PDF1.2 transcript accumulation. PDF1.2 transcript accumulation was monitored by qRT-PCR in seedlings from (D). Transcript accumulation was normalized to that of UBIQUITIN and is shown relative to the normalized transcript levels in the appropriate mock controls. Bars represent the $\log _{2}$ (mean) \pm SEM of biologically independent replicates. The letters above the bars indicate statistically significant differences (one-way ANOVA, $P=<0.05, F=14.93$, $\mathrm{DF}=7, \mathrm{n}=3$ for Col-0 and RNAi:LLP1-3, $\mathrm{n}=2$ for Ilp 1-1). 
2 Figure $4 L L P 1, L L P 2$, and/or $L L P 3$ dampen antagonistic SA-JA cross talk between defence pathways. (A) Exposure to salt drives up PR1 transcript levels when LLP1, LLP2, and/or LLP3 transcript levels are reduced. Four-week-old Col-0 wild type, Ilp11, and RNAi:LLP1-3 plants were irrigated with $300 \mathrm{mM} \mathrm{NaCl}$ three times over the course of 9 days. Three days later, $P R 1$ transcript accumulation in the leaves was determined by qRT-PCR. Transcript accumulation was normalized to that of UBIQUITIN and is shown relative to the normalized transcript levels in the appropriate mock controls. Bars represent the $\log _{2}$ (mean) \pm SEM of four biologically independent replicates. The letters above the bars indicate statistically significant differences (one-way ANOVA, $n=4, P<0.05, F=12.23$, $D F=11$ ). (B) In the absence of functional $L L P 1$, MeJA triggers SAR-like resistance in distal tissues. Col-0 wild type, IIp1-1, Ilp3, and RNAi:LLP1-3 plants were treated locally with $100 \mu \mathrm{M}$ MeJA by leaf infiltration. To monitor systemic SA-associated defence responses, leaves distal to the site of the initial treatment were inoculated with Pst 3 days after the primary treatment. Box plots represent average Pst titres in systemic leaves at 4 dpi from 4-5 biologically independent experiments, including 3-4 replicates each \pm min and max values. The letters above the box plots indicate statistically significant differences (Kruskal-Wallis test, $P<0.05, \mathrm{KW}$ test statistic=20.61, Col-0 mock $\mathrm{n}=17$, MeJA $\mathrm{n}=20$, IIp1-1 mock $\mathrm{n}=18$, MeJA $\mathrm{n}=19$, IIp3 mock $\mathrm{n}=11$, MeJA $\mathrm{n}=11$, RNAi:LLP1-3 mock $\mathrm{n}=19$, MeJA $\mathrm{n}=19$ ). (C) LLP3 promotes local SAR signal generation downstream of (Me)JA accumulating in the perimeter of HR (hypersensitive response) lesions. Elevated LLP3 expression promotes PDF1.2 expression and defence against necrotrophic pathogens through ERF1 (ETHYLENE RESPONSE FACTOR 1) as well as salt stress tolerance. In parallel with EDS1-dependent, SA-associated long distance signals, LLP3 promotes accumulation or transmission of a long distance SAR signal downstream of (Me)JA. Systemically, LLP1 balances incoming signals promoting SAR while restricting deleterious effects of SA-associated SAR on JAassociated defence responses. Abbreviations: NPR3/4, NON-EXPRESSOR OF PR GENES3/4 
B
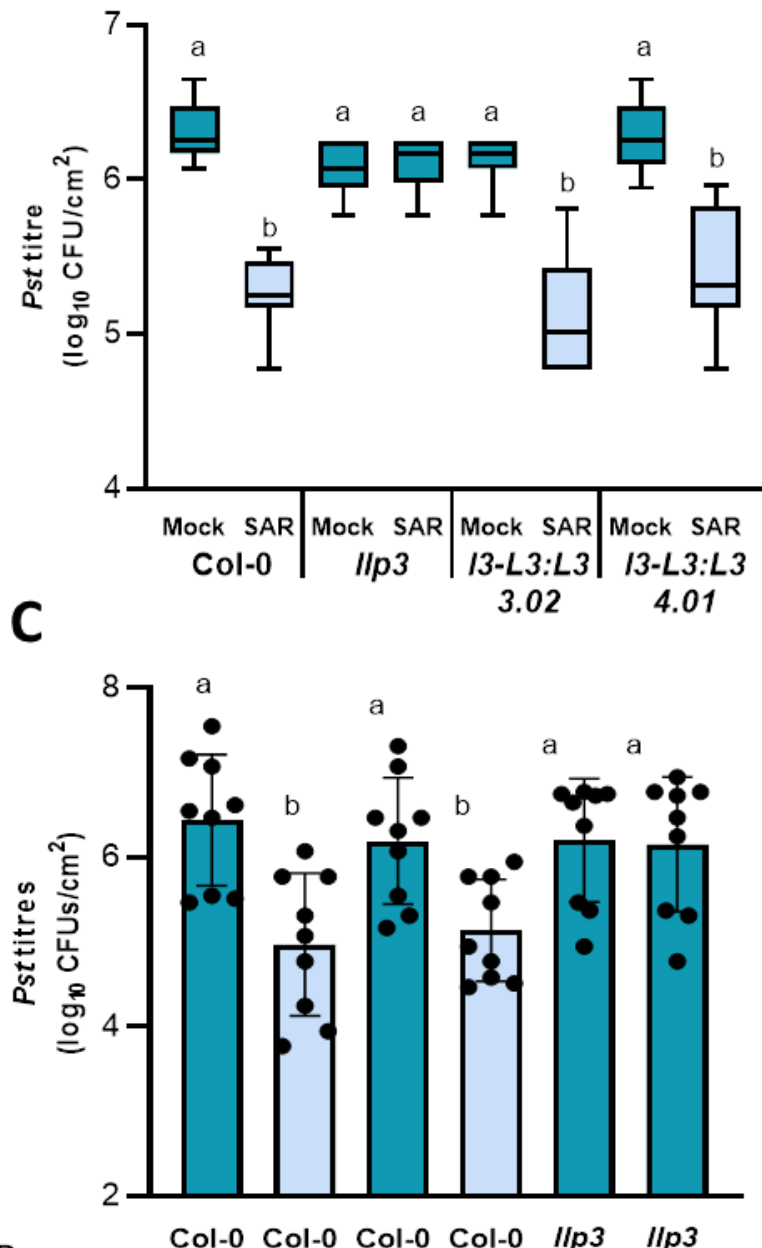

Donor:

Recipient:

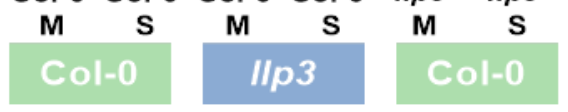

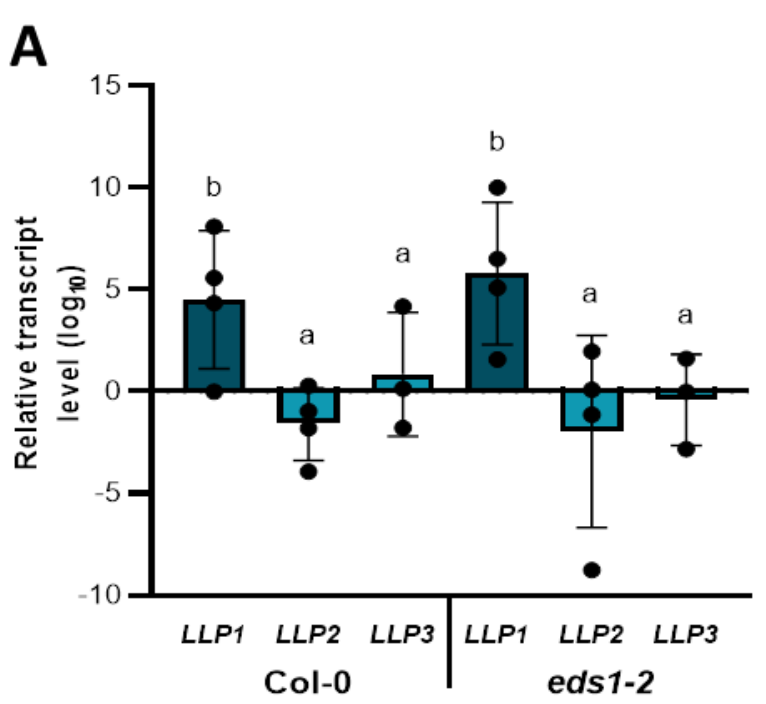

Figure 1 LEGUME LECTIN-LIKE PROTEIN3 (LLP3) promotes systemic acquired resistance (SAR) signal generation/transmission. (A) $L L P 3$ transcript accumulation is not affected by salicylic acid (SA). 4-week-old Col-0 wild type and eds1-2 Arabidopsis plants were spraytreated with $1 \mathrm{mM} \mathrm{SA}$, and 24 hours (h) later $L L P 1, L L P 2$, and $L L P 3$ transcript accumulation was determined by RT-qPCR. Transcript accumulation was normalized to that of UBIQUITIN and is shown relative to the normalized transcript levels in the appropriate mock controls. Bars represent the $\log _{2}$ (mean) \pm SEM of four biologically independent replicates. The letters above the bars indicate statistically significant differences (one-way ANOVA, $\mathrm{n}=4, \mathrm{P}<0.05, \mathrm{~F}=4.623, \mathrm{DF}=22$ ). (B) $\angle L P 3$ is required for SAR. Plants were infiltrated locally with either Pst AvrRpm1 (SAR) or with $10 \mathrm{mM} \mathrm{MgCl} 2$ as the mock control (M). To monitor SAR, 3 days after the primary treatment leaves distal to the initial treatment site were infiltrated with Pst. Plant lines included I/p3 mutants and 2 independently transformed complementation lines carrying a transgene driving $L L P 3$ expression from its native promoter (I3-L3:L3 3.02 and 4.01). Box plots represent average Pst titres in systemic leaves at 4 days postinoculation (dpi) from 4 biologically independent experiments, including 3 replicates each $\pm \min$ and max values. The letters above the box plots indicate statistically significant differences (Kruskal-Wallis test, $\mathrm{P}<0.05, \mathrm{n}=12, \mathrm{KW}$ statistic=101.4). (C) LLP3 is required to send, but not to receive phloemmobile SAR signals. Leaves of donor plants were inoculated with Pst AvrRpm1 (S) or with the appropriate mock control (M). After $24 \mathrm{~h}$, petiole exudates were collected from the donor plants and infiltrated into leaves of naïve recipient plants. 24h later, the treated leaves were challenged with Pst. Bars represent average Pst titres at $4 \mathrm{dpi}$ 43 from 3 biologically independent experiments, including 3 replicates each \pm SD. The letters above the bars indicate statistically significant differences (one-way ANOVA, $n=9, P<0.05, F=6.258, D F=35$ ). 


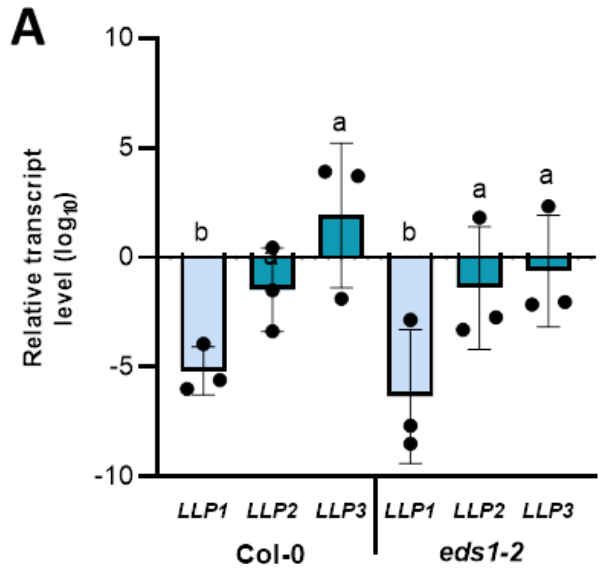

C

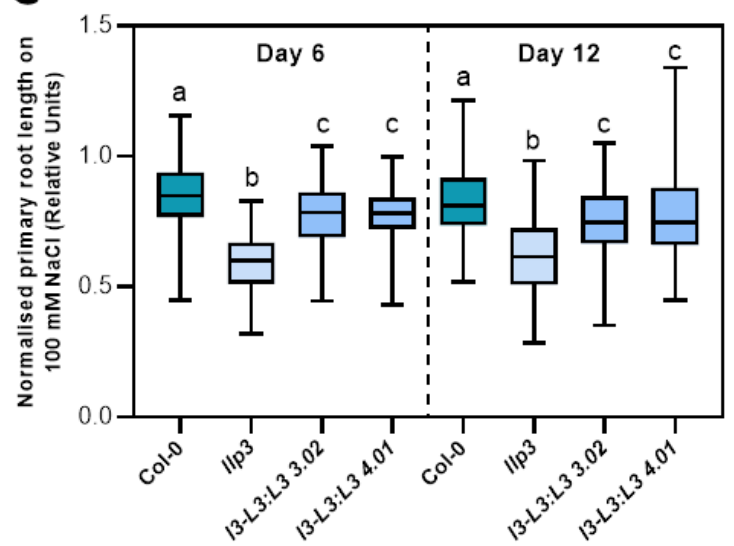

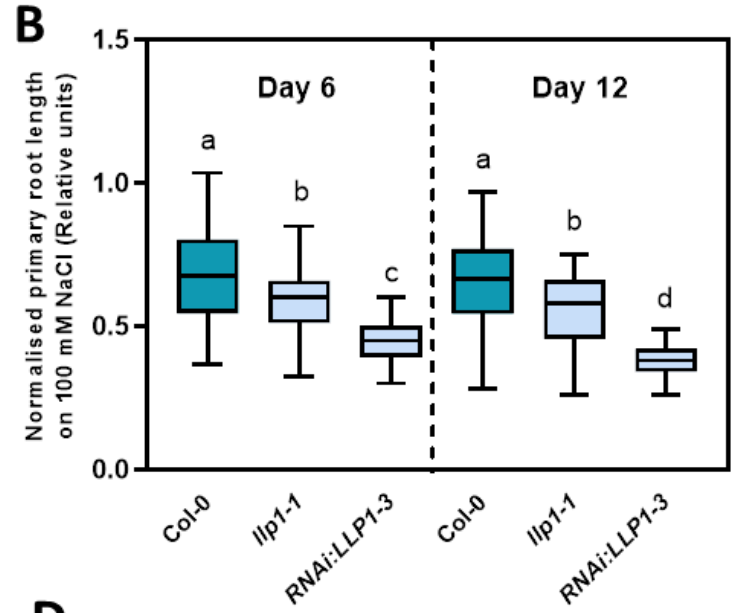

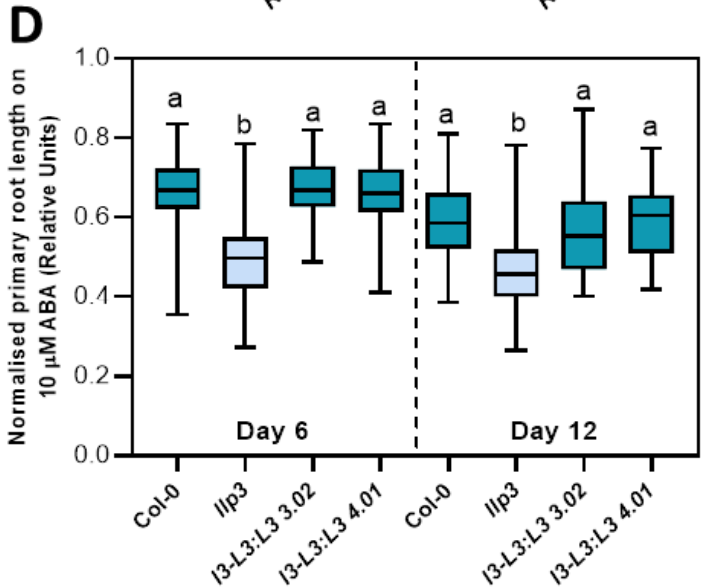

Figure $2 L L P 1, L L P 2$, and/or $L L P 3$ compromise Arabidopsis responses to salt stress. (A) $L L P 1$ transcript accumulation is reduced after ABA treatment. Col-0 wild type and eds1-2 plants were spray-treated with $100 \mu \mathrm{M}$ $\mathrm{ABA}$, and after $24 \mathrm{~h} L L P 1, L L P 2$, and/or LLP3 transcript accumulation was determined by RT-qPCR. Transcript accumulation was normalized to that of UBIQUITIN and is shown relative to the normalized transcript levels in the appropriate mock controls. Bars represent the $\log _{2}$ (mean) \pm SEM of three biologically independent replicates. The letters above the bars indicate statistically significant differences (one-way ANOVA, $\mathrm{n}=3, \mathrm{P}<0.05, \mathrm{~F}=6.291$, $\mathrm{DF}=40)$. (B/C) $L L P 1, L L P 2$, and/or $L L P 3$ compromise salt-associated root growth inhibition. Seedlings of Col-0 wild type, Ilp1-1, and RNAi:LLP1-3 (B) and of Ilp3 and two IIp3-LLP3:LLP3 complementation lines (I3-L3:L3 3.02 and 4.01 ; C) were germinated on control MS plates, and after 6 days transferred to either further control plates, or to plates supplemented with $100 \mathrm{mM} \mathrm{NaCl}$. Primary root length was measured at 6 and 12 days post transfer and normalized to that of the same genotype on control plates. Box plots represent average normalized root length \pm $\min$ and max values. The letters above the box plots indicate statistically significant differences (B: one-way ANOVA, $\mathrm{P}=<0.05, \mathrm{~F}=30.70$, $\mathrm{DF}=233$, for day 6 , Col-0 $\mathrm{n}=48$, $\| p 1-1 \mathrm{n}=38$, RNAi:LLP1 $\mathrm{n}=40$, for day $12 \mathrm{Col}-0 \mathrm{n}=48$, IIp1-1 $\mathrm{n}=29$, RNAi:LLP1-3 $\mathrm{n}=21$; C: Day 6: Kruskal-Wallis test, $\mathrm{P}=<0.05, \mathrm{KW}$ test statistic $=165.5$, Col-0 $\mathrm{n}=83$, Ilp3 $\mathrm{n}=86$, I3-L3:L3 $3.02 \mathrm{n}=96$, I3-L3:L3 4.01 n=89. Day 12: one-way ANOVA, $P=<0.05, F=25.08, D F=519, \mathrm{Col}-0$ $n=81$, IIp3 $n=84$, I3-L3:L3 $3.02 n=94$, I3-L3:L3 $4.01 n=84$ ). These experiments were repeated 3 (C) to 4-8 times (B) with comparable results. (D) LLP3 compromises root growth inhibition on $10 \mu \mathrm{M} \mathrm{ABA}$. Col-0 wild type, Ilp3 and two IIp3-LLP3:LLP3 complementation lines were treated as described in $(\mathrm{B} / \mathrm{C})$, and the treatment plates were supplemented with $10 \mu \mathrm{M}$ ABA. Box plots represent average normalized root length \pm min and max values. The letters above the box plots indicate statistically significant differences (Day 6: one-way ANOVA, $P=<0.05$, $F=76.10$, DF=538, Col-0 n=76, IIp3 n=86, 3.01 n=89, I3-L3:L3 3.02 n=98, I3-L3:L3 4.01 n=94, 8.01 n=96. Day 12: Kruskal-Wallis test, $P=<0.05, \mathrm{KW}$ test statistic $=121.1$, Col-0 $n=85$, IIp3 $n=90$, I3-L3:L3 3.02 $n=100$, I3-L3:L3 4.01 $\mathrm{n}=97$ ). This experiment was repeated 3 times with comparable results. 


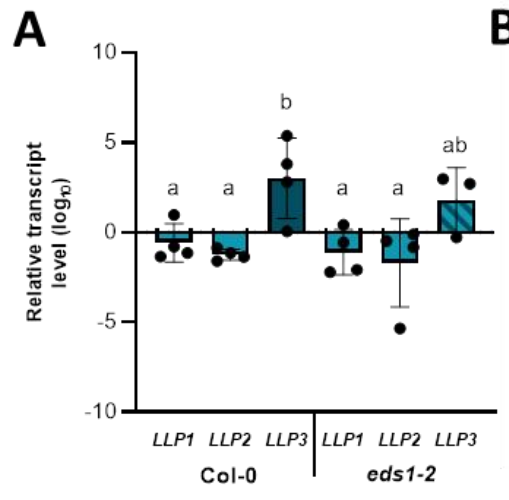

D

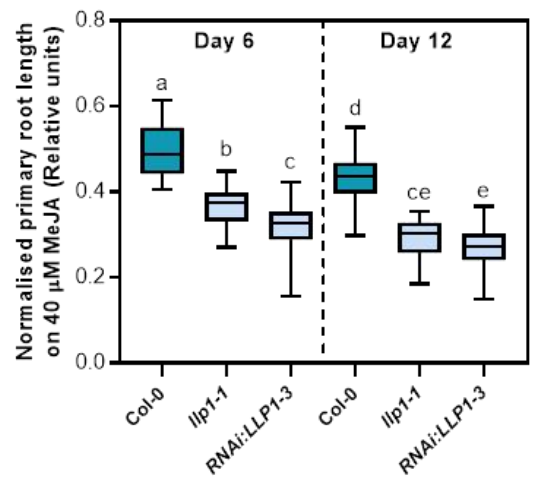

B

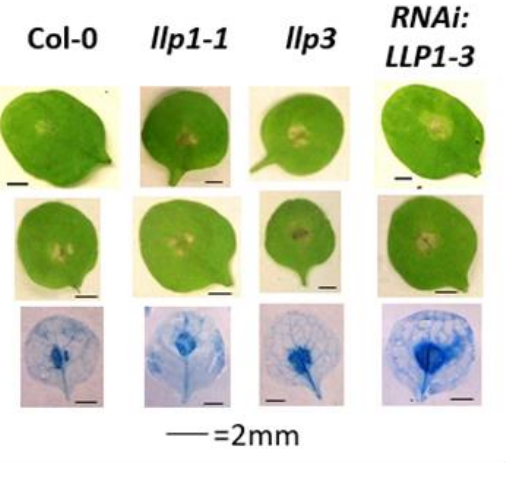

E

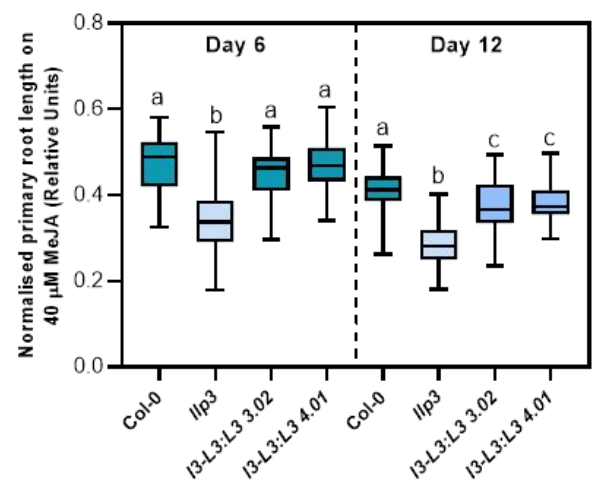

C
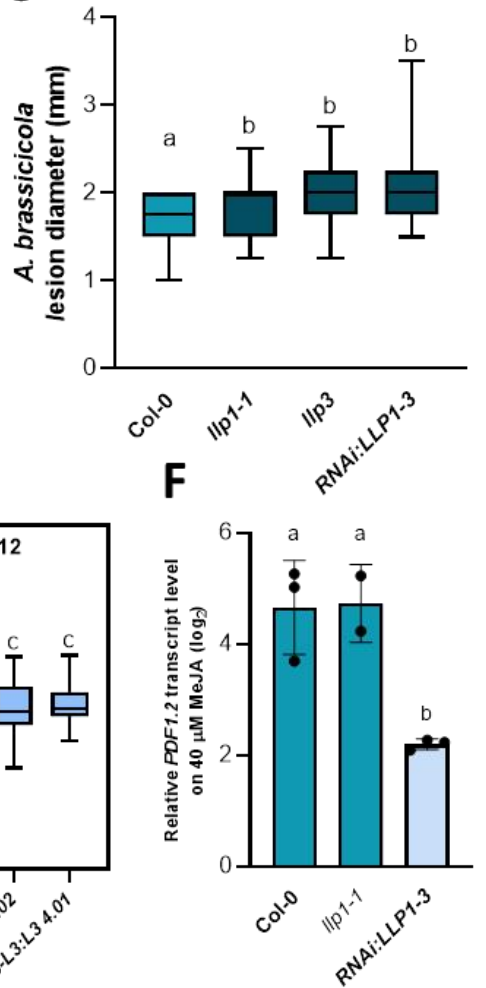

Figure $3 L L P 1, L L P 2$, and/or $L L P 3$ differentially affect jasmonic acid (JA)-associated responses in Arabidopsis. (A) LLP3 transcript accumulation is induced by methyl jasmonate (MeJA). Col-0 wild type and eds 1-2 plants were spray-treated with $100 \mu \mathrm{M} \mathrm{MeJA}$, and after $24 \mathrm{~h}$ LLP1, LLP2, and/or LLP3 transcript accumulation was determined by RT-qPCR. Transcript accumulation was normalized to that of UBIQUITIN and is shown relative to the normalized transcript levels in the appropriate mock controls. Bars represent the $\log _{2}($ mean $) \pm S E M$ of four biologically independent replicates. The letters above the bars indicate statistically significant differences (oneway ANOVA, $\mathrm{n}=4, \mathrm{P}<0.05, \mathrm{~F}=4.493, \mathrm{DF}=45)$. (B/C) $L L P 1, L L P 2$, and/or $L L P 3$ promote JA-associated defence against Alternaria brassicicola. Droplets containing spores of the necrotrophic fungus $A$. brassicicola were placed on the leaves of four-week-old Col-0 wild type, Ilp1-1, IIp3, and RNAi:LLP1-3 plants. Resulting lesions were photographed (B) and measured (C) 5 days later. Box plots in (C) represent mean lesion diameters from 4 biologically independent experiments including 15 replicates each \pm min and max values. The letters above the box plots indicate statistically significant differences (Kruskal-Wallis test, $P=<0.05$, KW test statistic $=24.10, n=60$ for all genotypes). (D/E) LLP1, LLP2, and/or LLP3 compromise JA-associated root growth inhibition. Seedlings of Col-0 wild type, Ilp1-1, and RNAi:LLP1-3 (D) and of I/p3 and two IIp3-LLP3:LLP3 complementation lines (I3-L3:L3 3.02 and 4.01 ; E) were germinated on control MS plates, and after 6 days transferred to either further control plates, or to plates supplemented with $40 \mu \mathrm{M}$ MeJA. Primary root length was measured at 6 and 12 days post transfer and normalized to that of the same genotype on control plates. Box plots represent average normalized root length $\pm \min$ and max values. The letters above the box plots indicate statistically significant differences (D: Day 6: one-way ANOVA, $F=44.87, D F=147$, Col-0 $n=29$, Ilp1-1, RNAi:LLP1-3 $n=30$. Day 12: one-way ANOVA, $\mathrm{F}=74.62, \mathrm{DF}=175$, Col-0 $\mathrm{n}=29$, Ilp1-1 $\mathrm{n}=30, \mathrm{RNAi}: L L P 1-3 \mathrm{n}=28$; $\mathrm{E}$ : Day 6: one-way ANOVA, $\mathrm{P}<0.05, \mathrm{~F}=61.40$, $\mathrm{DF}=541$, Col-0 $n=71$, IIp3 $n=85$, I3-L3:L3 3.02 $n=94$, I3-L3:L3 4.01 $n=97$. Day 12: Kruskal-Wallis test, $P=<0.05$, $\mathrm{KW}$ test statistic $=140.7$, Col-0 $n=63$, IIp3 $n=73$, I3-L3:L3 $3.02 n=94$, I3-L3:L3 $4.01 n=97,8.01 n=95)$. These experiments were repeated $3(E)$ to 5 times (D) with comparable results. (F) LLP1, LLP2, and/or LLP3 compromise MeJA-induced PDF1.2 transcript accumulation. PDF1.2 transcript accumulation was monitored by qRT-PCR in seedlings from (D). Transcript accumulation was normalized to that of UBIQUITIN and is shown relative to the normalized transcript levels in the appropriate mock controls. Bars represent the $\log _{2}($ mean $) \pm S E M$ of biologically independent replicates. The letters above the bars indicate statistically significant differences (oneway ANOVA, $\mathrm{P}=<0.05, \mathrm{~F}=14.93, \mathrm{DF}=7, \mathrm{n}=3$ for Col-0 and RNAi:LLP1-3, $\mathrm{n}=2$ for $\| \mathrm{p} 1-1$ ). 
A

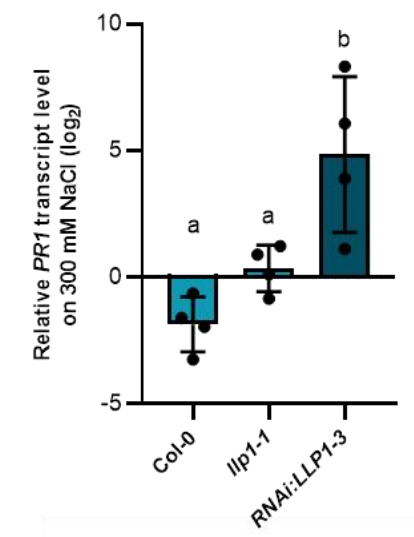

B

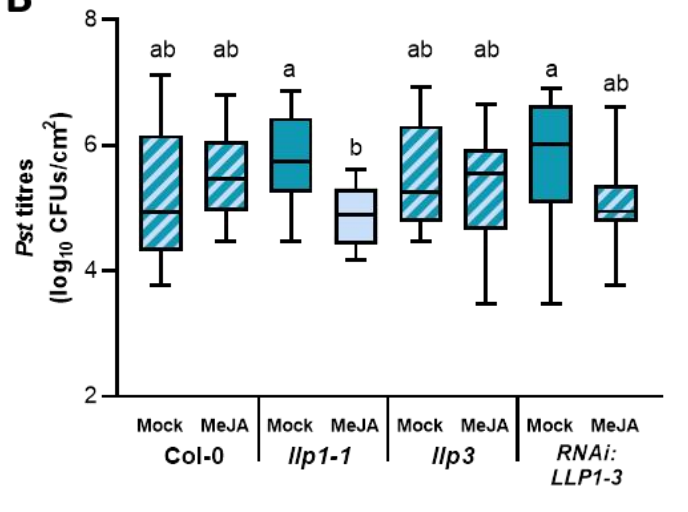

C

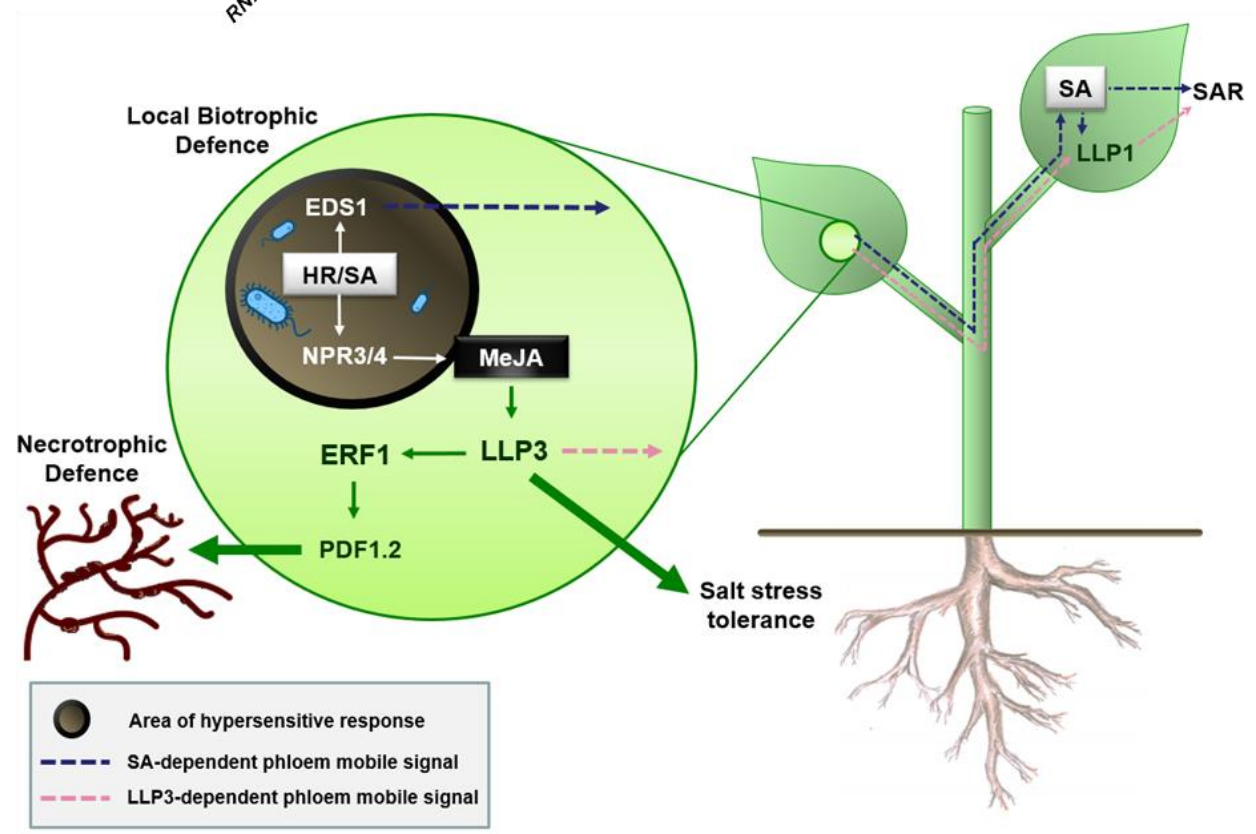

Figure $4 L L P 1, L L P 2$, and/or $L L P 3$ dampen antagonistic SA-JA cross talk between defence pathways. (A) Exposure to salt drives up $P R 1$ transcript levels when $L L P 1, L L P 2$, and/or $L L P 3$ transcript levels are reduced. Four-week-old Col-0 wild type, Ilp1-1, and RNAi:LLP1-3 plants were irrigated with $300 \mathrm{mM} \mathrm{NaCl}$ three times over the course of 9 days. Three days later, $P R 1$ transcript accumulation in the leaves was determined by qRT-PCR. Transcript accumulation was normalized to that of UBIQUITIN and is shown relative to the normalized transcript levels in the appropriate mock controls. Bars represent the $\log _{2}($ mean) \pm SEM of four biologically independent replicates. The letters above the bars indicate statistically significant differences (one-way ANOVA, $n=4, P<0.05$, $\mathrm{F}=12.23, \mathrm{DF}=11$ ). (B) In the absence of functional LLP1, MeJA triggers SAR-like resistance in distal tissues. Col-0 wild type, IIp1-1, I/p3, and RNAi:LLP1-3 plants were treated locally with $100 \mu \mathrm{M}$ MeJA by leaf infiltration. To monitor systemic SA-associated defence responses, leaves distal to the site of the initial treatment were inoculated with Pst 3 days after the primary treatment. Box plots represent average Pst titres in systemic leaves at 4 dpi from 4-5 biologically independent experiments, including 3-4 replicates each \pm min and max values. The letters above the box plots indicate statistically significant differences (Kruskal-Wallis test, $P<0.05, \mathrm{KW}$ test statistic=20.61, Col-0 mock $n=17$, MeJA $n=20$, Ilp1-1 mock $n=18$, MeJA $n=19$, Ilp3 mock $n=11$, MeJA $n=11$, RNAi:LLP1-3 mock $n=19$, MeJA $n=19$ ). (C) LLP3 promotes local SAR signal generation downstream of (Me)JA accumulating in the perimeter of HR (hypersensitive response) lesions. Elevated LLP3 expression promotes PDF1.2 expression and defence against necrotrophic pathogens through ERF1 (ETHYLENE RESPONSE FACTOR 1) as well as salt stress tolerance. In parallel with EDS1-dependent, SA-associated long distance signals, LLP3 promotes accumulation or transmission of a long distance SAR signal downstream of (Me)JA. Systemically, LLP1 balances incoming signals promoting SAR while restricting deleterious effects of SAassociated SAR on JA-associated defence responses. Abbreviations: NPR3/4, NON-EXPRESSOR OF PR GENES3/4 